\title{
Latent Fingerprint Matching using Descriptor-based Hough Transform
}

\author{
Alessandra A. Paulino, Jianjiang Feng, Member, IEEE, and Anil K. Jain, Fellow, IEEE
}

\begin{abstract}
Identifying suspects based on impressions of fingers lifted from crime scenes (latent prints) is a routine procedure that is extremely important to forensics and law enforcement agencies. Latents are partial fingerprints that are usually smudgy, with small area and containing large distortion. Due to these characteristics, latents have a significantly smaller number of minutiae points compared to full (rolled or plain) fingerprints. The small number of minutiae and the noise characteristic of latents make it extremely difficult to automatically match latents to their mated full prints that are stored in law enforcement databases. Although a number of algorithms for matching full to full fingerprints have been published in the literature, they do not perform well on the latent to full matching problem. Further, they often rely on features that are not easy to extract from poor quality latents. In this paper, we propose a new fingerprint matching algorithm which is especially designed for matching latents. The proposed algorithm uses a robust alignment algorithm (descriptor-based Hough transform) to align fingerprints and measures similarity between fingerprints by considering both minutiae and orientation field information. To be consistent with the common practice in latent matching (i.e. only minutiae are marked by latent examiners), the orientation field is reconstructed from minutiae. Since the proposed algorithm relies only on manually marked minutiae, it can be easily used in the law enforcement applications. Experimental results on two different latent databases (NIST SD27 and WVU latent databases) show that the proposed algorithm outperforms two well optimized commercial fingerprint matchers. Further, a fusion of the proposed algorithm and commercial fingerprint matchers leads to improved matching accuracy.
\end{abstract}

Index Terms-Fingerprints, latents, matching, local descriptors, Minutia Cylinder Code, Hough transform.

\section{INTRODUCTION}

$\mathbf{L}$ Aw enforcement agencies have started using fingerprint recognition technology to identify suspects since the early 20th century [2]. Nowadays, automated fingerprint identification system (AFIS) has become an indispensable tool for law enforcement agencies.

A. A. Paulino and A. K. Jain are with the Department of Computer Science and Engineering, Michigan State University, East Lansing, MI, 48824 USA. A. $\mathrm{K}$. Jain is also with the Department of Brain and Cognitive Engineering, Korea University, Anamdong, Seongbukgu, Seoul 136-713, Republic of Korea. Email: \{paulinoa, jain\}@cse.msu.edu. A. K. Jain is the corresponding author.

J. Feng is with the Department of Automation, Tsinghua University, Beijing, China. E-mail: jfeng@tsinghua.edu.cn.

A. K. Jain's research was partially supported by WCU (World Class University) program funded by the Ministry of Education, Science and Technology through the National Research Foundation of Korea (R31-10008). A. A. Paulino's research was supported by the Fulbright Program (A15087649) and Brazilian Government through a CAPES Foundation/Ministry of Education grant (1667-07-6)

An early version of this paper appeared in the proceedings of the International Joint Conference on Biometrics (IJCB) 2011 [1].

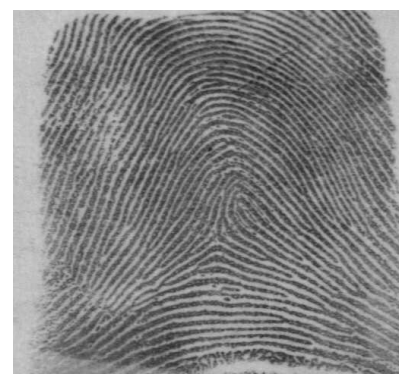

(a) Rolled

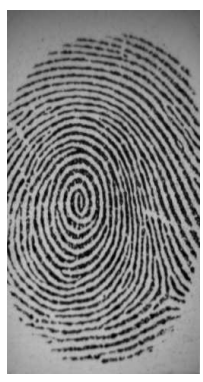

(b) Plain

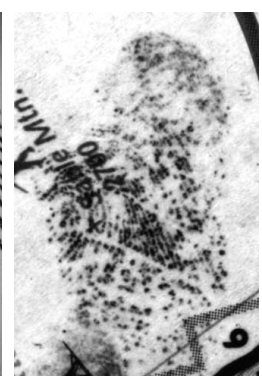

(c) Latent
Fig. 1. Three types of fingerprint impressions. Rolled and plain fingerprints are also called full fingerprints.

There are essentially three types of fingerprints in law enforcement applications (see Fig. 1): (i) rolled, which is obtained by rolling the finger "nail-to-nail" either on a paper (in this case ink is first applied to the finger surface) or the platen of a scanner; (ii) plain, which is obtained by placing the finger flat on a paper or the platen of a scanner without rolling; and (iii) latents, which are lifted from surfaces of objects that are inadvertently touched or handled by a person typically at crime scenes. Lifting of latents may involve a complicated process, and it can range from simply photographing the print to more complex dusting or chemical processing [2].

Rolled prints contain the largest amount of information about the ridge structure on a fingerprint since they capture the largest finger surface area; latents usually contain the least amount of information for matching or identification because of their size and inherent noise. Compared to rolled or plain fingerprints, latents are smudgy and blurred, capture only a small finger area, and have large nonlinear distortion due to pressure variations. Due to their poor quality and small area, latents have a significantly smaller number of minutiae compared to rolled or plain prints (the average number of minutiae in NIST Special Database 27 (NIST SD27) [3] images is 21 for latents versus 106 for their mated rolled prints). These characteristics make the latent fingerprint matching problem very challenging.

Fingerprint examiners who perform manual latent fingerprint identification follow a procedure referred to as ACE$\mathrm{V}$ (analysis, comparison, evaluation and verification) [4]. Because the ACE- $\mathrm{V}$ procedure is quite tedious and time consuming for latent examiners, latents are usually matched against full prints of a small number of suspects identified by other means, such as eye witness description or M.O. (mode of operation). With the availability of AFIS, fingerprint examiners are able to match latents against a large fingerprint 
database using a semi-automatic procedure that consists of following stages: (i) manually mark the features (minutiae and singular points) in the latent, (ii) launch an AFIS search, and (iii) visually verify the top- $N$ ( $N$ is typically 50$)$ candidate fingerprints returned by AFIS. The accuracy and speed of this latent matching procedure is still not satisfactory. It certainly does not meet the "lights-out mode" of operation desired by the FBI and included in the Next Generation Identification [5].

For fingerprint matching, there are two major problems which need to be solved. The first is to align the two fingerprints to be compared and the second is to compute a match score between the two fingerprints. Alignment between a latent and a rolled print is a challenging problem because latents often contain a small number of minutiae and undergo large skin distortion. To deal with these two problems, we propose the descriptor-based Hough transform (DBHT), which is a combination of the generalized Hough transform and a local minutiae descriptor, called Minutia Cylinder Code (MCC) [6]. The MCC descriptor improves the distinctiveness of minutiae while the Hough transform method can accumulate evidence as well as improve the robustness against distortion. Match score computation between a latent and a rolled print is also challenging because the number of mated minutiae is usually small. To address this issue, we further consider orientation field as a factor in computing match score. Since we only have manually marked minutiae for latents, a reconstruction algorithm is used to obtain orientation field from minutiae.

The proposed matcher was tested on two latent fingerprint databases, NIST SD27 database and West Virginia University latent fingerprint database (WVU LFD). Two COTS matchers and a state-of-the-art non-commercial fingerprint matching algorithm (MCC SDK) were also evaluated on the same databases. Our algorithm was found to perform better than the other three matchers being compared on both the databases. Extensive experiments on fusion of matchers and effect of fingerprint quality were also conducted.

The rest of the paper is organized as follows: in Section II, related work is reviewed; in Section III, all steps of our proposed method are described; in Section IV, our experimental results are presented and discussed; in Section V, we present our conclusions and future work.

\section{RELATED WORK}

In this section, we review related work in four areas: published research on full fingerprint matching ${ }^{1}$, published research on latent fingerprint matching, evaluation of latent fingerprint technologies (ELFT), and evaluation of latent examiners.

\section{A. Full Fingerprint Matching}

The majority of the algorithms developed for fingerprint matching are based on minutiae. Although minutiae carry a great amount of discriminatory information, in some cases additional features may help increase the accuracy. Most

\footnotetext{
${ }^{1}$ See Chapter 4 in [7] for a more comprehensive review of this topic.
}

proposed algorithms for fingerprint matching that use nonminutiae features also use minutiae. For example, some algorithms combine ridge orientation with minutiae information either at feature level by including ridge orientation information in local minutiae descriptors [8], [9] or at score level by combining scores from minutiae matching and global orientation field matching [9], [10].

Several recent studies on fingerprint matching have focused on the use of local minutiae descriptors [6], [8], [9], [11], [12], [13], [14]. In most of these studies, the initial step consists of using local minutiae descriptors to obtain the alignment between two fingerprints by considering the most similar minutiae pair; then, a global consolidation step is performed to obtain a better matching performance. Since these algorithms are usually tuned and evaluated using FVC databases (plain fingerprints) or NIST Special Database 4 (rolled fingerprints), their performances on latent fingerprints are unknown.

\section{B. Latent Fingerprint Matching}

Recent research and development efforts on latent fingerprints can be classified into three streams according to the manual input required from fingerprint examiners: consistent with existing practice, increasing manual input, or reducing manual input. Because of large variations in latent fingerprint quality and specific requirements of practical applications (crime scenes, border crossing points, battle fields), each of the three streams has its value.

Improved latent matching accuracy has been reported by using extended features, which are manually marked for latents [15], [16], [17], [18]. However, marking extended features (orientation field, ridge skeleton, etc.) in poor quality latents is very time-consuming and might be only feasible in rare cases. Thus, some studies have concentrated on latent matching using a reduced amount of manual input, such as manually marked region of interest (ROI) and singular points [19], [20]. However, only a small portion of latents can be correctly identified using this approach. Hence our proposed matcher takes manually marked minutiae as input and, therefore, it is consistent with existing practice. There have also been some studies on fusion of multiple matchers [21] or multiple latent prints [22].

\section{Evaluation of Latent Fingerprint Technologies}

NIST has been conducting a multi-phase project on Evaluation of Latent Fingerprint Technologies (ELFT) to evaluate latent feature extraction and matching techniques [23]. Since all participating algorithms in ELFT are proprietary, we have no information on the details of these algorithms. The purpose of ELFT-Phase I was to assess the feasibility of latent fingerprint identification systems using Automated Feature Extraction and Matching (AFEM), while the purpose of ELFT-Phase II was to actually measure the performance of state-of-the-art AFEM technology and evaluate whether it was viable to have those systems in the operational use to reduce the amount of time needed by latent examiners to manually mark latents thereby increasing the throughput. 


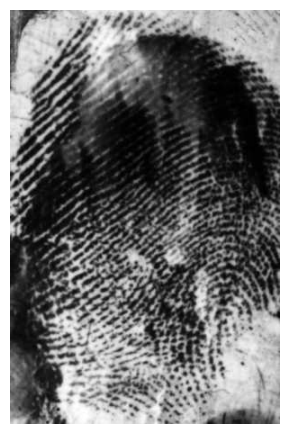

(a) Good

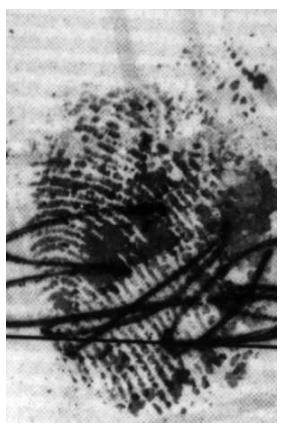

(b) Bad

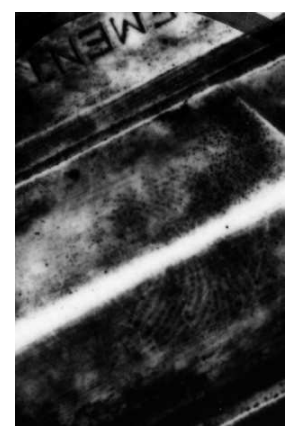

(c) Ugly
Fig. 2. Latent fingerprints of three different quality levels in NIST SD27.

In Phase I, latent images were selected from both operational and non-operational scenarios. The most accurate system showed a rank-1 accuracy of $80 \%$ (100 latents against 10,000 rolled prints) [24]. In Phase II, latent images were selected from only operational environments. The rank-1 accuracy of the most accurate system was $97.2 \%$ (835 latents against 100, 000 rolled prints) [25]. These accuracies cannot be directly compared since the Phase I and Phase II evaluations used different latent databases. Furthermore, the quality of latents used in Phase II is better compared to Phase I. As shown in Fig. 2, the quality of latents varies significantly.

The impressive matching accuracy reported in ELFT does not support that the current practice of manually marking minutiae in latents should be changed. Although latents in Phase II were selected from operational scenarios, they represent successful identifications in actual case examinations using existing AFIS technology. In the ACE-V process, when the examiner analyzes the latent image he/she decides whether the latent has value for exclusion only, value for individualization or no value. If a latent is classified as of no value, no comparison is performed. If the latent is classified in one of the other two categories, then comparisons are performed and the examiners can make an individualization, an exclusion, or determine the comparison to be inconclusive. So the latents which are successfully identified constitute only a small part of all latents, which are of reasonable quality. For this reason, in the ELFT-Phase II report [25] the authors concluded that only a limited class of latents can benefit from AFEM technology.

NIST has conducted another evaluation of latent fingerprint technologies using extended feature sets manually marked by latent examiners [26]. In this evaluation, the purpose was to investigate the matching accuracy when (i) latent images and/or (ii) sets of manually marked features were provided. This evaluation suggested that the highest accuracy was obtained when the input included both the latent image and manually marked features.

\section{Evaluation of Latent Examiners}

A latent examiner can be viewed as a slow but very accurate "matcher". Because they are much slower than automatic matchers, quantitatively estimating the accuracy of latent examiners is not easy. Hence the numbers of fingerprint pairs used in several "black box" tests of latent examiners are not large [27], [28], [29]. Although the exact numbers reported in these studies may not reflect the real practice, the qualitative conclusions are very useful. It was found that latent examiners's conclusion are not always in agreement, especially in the case of poor quality latents [27]. In addition, the same examiner can change his/her conclusions on the same fingerprint pair at a later time [28]. These inconsistences may increase under bias [29].

These issues associated with including latent examiners in the latent identification process will only be solved when the automatic matcher can outperform latent examiners in accuracy. No matter how successful the application of automatic fingerprint recognition technology might be, we cannot say fingerprint matching is a "solved problem" before we can reach the goal of outperforming latent examiners.

\section{LATENT MATCHING APPROACH}

Given a latent fingerprint (with manually marked minutiae) and a rolled fingerprint, we extract additional features from both prints, align them in the same coordinate system, and compute a match score between them. These three steps are described in the following subsections. An overview of the proposed algorithm is shown in Fig. 3.

\section{A. Feature Extraction}

The proposed matching approach uses minutiae and orientation field from both latent and rolled prints. Minutiae are manually marked by latent examiners in the latent, and automatically extracted using commercial matchers in the rolled print. Based on minutiae, local minutiae descriptors are built and used in the proposed descriptor-based alignment and scoring algorithms. Orientation field is reconstructed from minutiae location and direction for the latents as proposed in [30], and orientation field is automatically extracted from the rolled print images by using a gradient-based method. Local minutia descriptors and orientation field reconstruction are presented in the following subsections.

1) Local Minutia Descriptor: Local descriptors have been widely used in fingerprint matching (e.g. [11], [8], [12], [18], [6]). Feng and Zhou [31] evaluated the performance of local descriptors associated with fingerprint matching in four categories of fingerprints: good quality, poor quality, small common region, and large plastic distortion. They also coarsely classified the local descriptors as image-based, texture-based, and minutiae-based descriptors. Their results show that the minutiae-based descriptor, Minutia Cylinder Code (MCC) [6], performs better in three of the four categories, and texturebased descriptor performs better for the small common region category.

A minutia cylinder records the neighborhood information of a minutia as a 3D function. A cylinder contains several layers and each layer represents the density of neighboring minutiae along the corresponding direction. The cylinder can be concatenated as a vector, and therefore the similarity between two minutia cylinders can be efficiently computed. Fig. 4b shows the sections of two valid cylinders associated with the two corresponding minutiae (in the latent and in the 


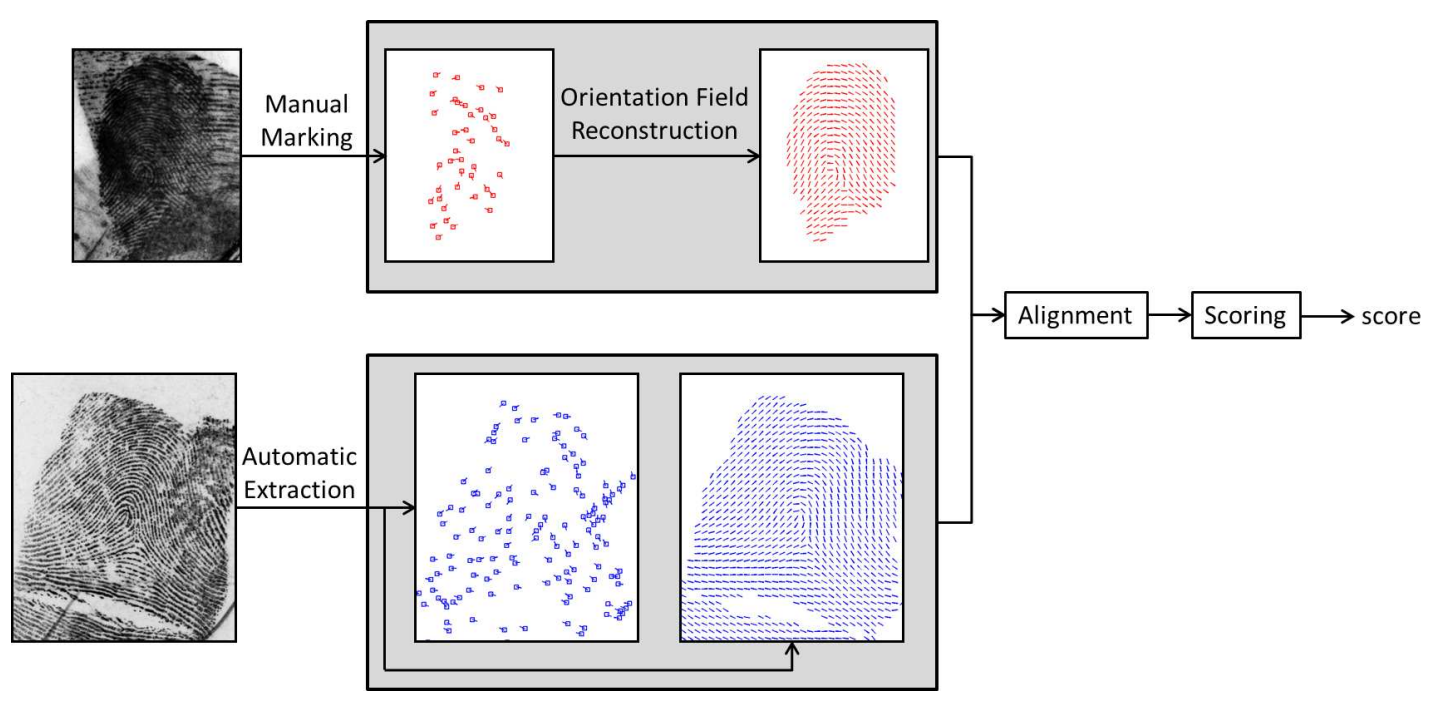

Fig. 3. Overview of the proposed approach.

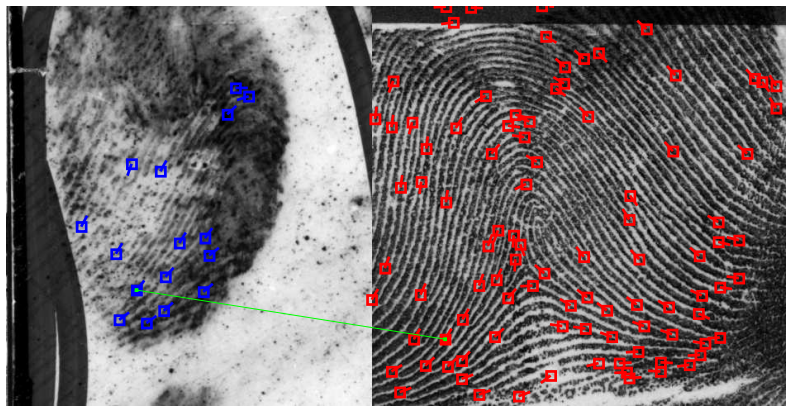

(a) Latent and corresponding rolled print with a mated minutiae pair indicated.
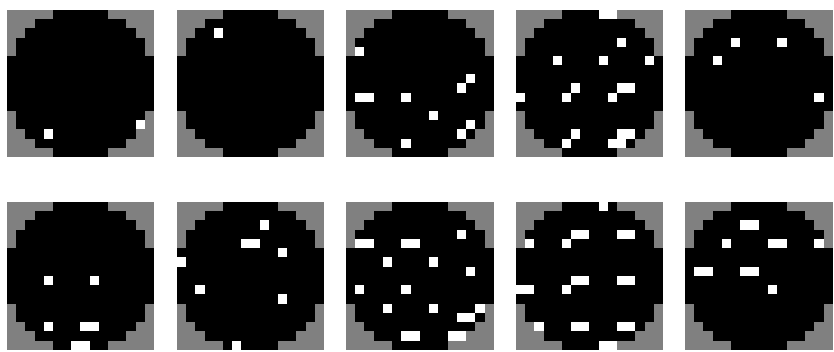

(b) Sections of the cylinder corresponding to the minutia indicated in the latent (first row) and in the rolled print (second row).

Fig. 4. Sections of two cylinders associated with the two corresponding minutiae, one in latent and other in rolled print.

rolled print) indicated in Fig. 4a. A more detailed description of the cylinder generation and of the similarity between two cylinders can be found in [6].

2) Orientation Field Reconstruction: Orientation field can be used in several ways to improve fingerprint matching performance, such as by matching orientation fields directly and fusing scores with other matching scores, or by enhancing the images to extract more reliable features. Orientation field estimation using gradient-based method is very reliable in good quality images [7]. However, when the image contains noise, this estimation becomes very challenging. A few model-
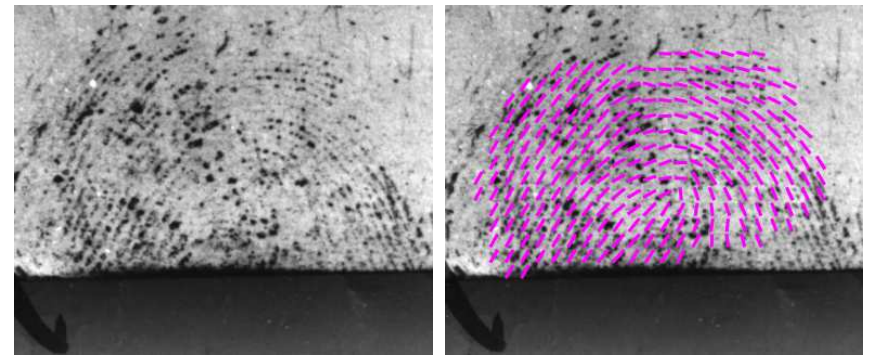

Fig. 5. A latent fingerprint in NIST SD27 and the reconstructed orientation field overlaid on the latent.

based orientation field estimation methods have been proposed ([32], [33], [34]) that use singular points as input to the model. In the latent fingerprint matching case, it is very challenging to estimate the orientation field based only on the image due to the poor quality and small area of the latent. Moreover, if singular points are to be used, they need to be manually marked (and they are not always present) in the latent fingerprint image.

Hence, we use a minutiae-based orientation field reconstruction algorithm proposed in [30] which takes manually marked minutiae in latents as input and outputs an orientation field. This approach estimates the local ridge orientation in a block by averaging the direction of neighboring minutiae. The orientation field is reconstructed only inside the convex hull of minutiae. Since the direction of manually marked minutiae is very reliable, the orientation field reconstructed using this approach is quite accurate except in areas absent of minutiae or very close to singular points (see Fig. 5 for an example). For rolled fingerprints, orientation field is automatically extracted using a gradient-based method [7].

\section{B. Alignment}

Fingerprint alignment or registration consists of estimating the parameters (rotation and translation) that align two fingerprints. There are a number of features that may be 
used to estimate alignment parameters between two fingerprints, including singular points, orientation field, ridges, and minutiae. There are also a number of methods to align two fingerprints: Generalized Hough Transform, local descriptors, energy minimization, etc $^{2}$.

In the latent matching case, singularities are not always present in latents, making it difficult to base the alignment of the fingerprint on singular points alone. To obtain manually marked orientation field is expensive, and to automatically extract orientation field from a latent image is a very challenging problem. Since manually marking minutiae is a common practice for latent matching, our approach to align two fingerprints is based on minutiae.

Local descriptors can also be used to align two fingerprints. In this case, usually the most similar minutiae pair is used as a base for the transformation parameters (rotation and translation), and the most similar pair is chosen based on a measure of similarity between the local descriptors of the minutiae pair.

Ratha et al. introduced an alignment method for minutiae matching that estimates rotation, scale, and translation parameters using a Generalized Hough Transform [35]. Given two sets of points (minutiae), a matching score is computed for each transformation in the discretized set of all allowed transformations. For each pair of minutiae, one minutia from each image (latent or full), and for given scale and rotation parameters, unique translation parameters can be computed. Each parameter receives "a vote" that is proportional to the matching score for the corresponding transformation. The transformation that gives the maximum score is considered the best one. In our approach, the alignment is conducted in a similar way, but the evidence for each parameter is accumulated based on the similarity between the local descriptors of the two involved minutiae, with the similarity and descriptor being the ones described in Section III-A1.

The descriptor-based Hough transform alignment algorithm takes as input two sets of minutiae, $M_{\mathrm{L}}$ and $M_{\mathrm{R}}$, and two sets of local descriptors $C_{\mathrm{L}}$ and $C_{\mathrm{R}}$, one set corresponding to the latent and one to the rolled print. Each set contains a local descriptor for each minutia. A high level algorithm of the proposed approach to align two fingerprints given the sets of minutiae and of local descriptors is shown in Algorithm 1.

Given two sets of minutiae, one from the latent and the other from the rolled print being compared, translation and rotation parameters can be obtained for each possible minutiae pair (one minutia from each set). Let $\left\{\left(x_{l}, y_{l}, \theta_{l}\right)\right\}$ and $\left\{\left(x_{r}, y_{r}, \theta_{r}\right)\right\}$ be the minutiae sets for latent and rolled prints, respectively, centered at their means. Then, for each pair of minutiae, we have

$$
\begin{gathered}
\Delta \theta=\min \left(\left\|\theta_{l}-\theta_{r}\right\|, 360-\left\|\theta_{l}-\theta_{r}\right\|\right), \\
\left(\begin{array}{c}
\Delta x \\
\Delta y
\end{array}\right)=\left(\begin{array}{c}
x_{r} \\
y_{r}
\end{array}\right)-\left(\begin{array}{cc}
\cos \Delta \theta & \sin \Delta \theta \\
-\sin \Delta \theta & \cos \Delta \theta
\end{array}\right)\left(\begin{array}{c}
x_{l} \\
y_{l}
\end{array}\right) .
\end{gathered}
$$

Since the scale (resolution) is fixed in fingerprint matching, unique translation parameters can be obtained for each pair based on the rotation difference between the two minutiae in

\footnotetext{
${ }^{2}$ Refer to Chapter 4 of [7] for details and published work.
}

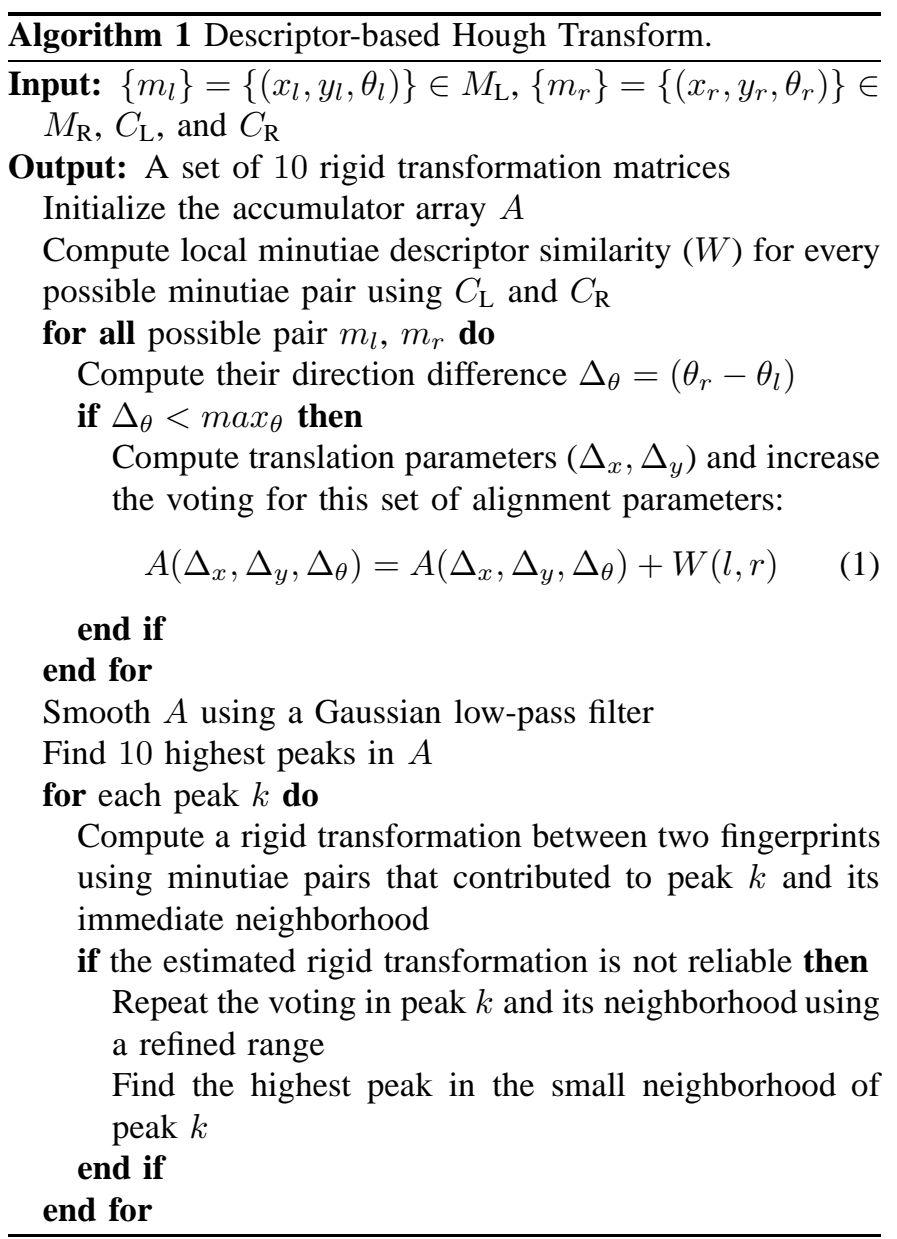

the pair. The translation and rotation parameters need to be quantized to the closest bins. After the quantization, evidence is accumulated in the corresponding bin based on the similarity between the local minutiae descriptors. The assumption here is that true mated minutiae pairs will vote for very similar sets of alignment parameters, while non-mated minutiae pairs will vote randomly throughout the parameter space. As a result, the set of parameters that presents the highest evidence is considered the best one. For robustness, ten sets of alignment parameters with strong evidence are considered.

In order to make the alignment computationally efficient and also more accurate, we use a two-step approach to compute the alignment parameters for a fingerprint pair. The first step is to perform the voting using the Descriptor-based Hough Transform. If the bins are too small, the true peak in the Hough Transform space cannot receive sufficient votes. On the other hand, if the bins are too large, they will not provide accurate alignment parameters. The strategy we adopted is to keep the bins relatively large, and to include a second step to compute reliable alignment parameters. This second step consists of using the minutiae pairs that vote for a peak to compute a rigid transformation between the two fingerprints. The use of voting minutiae pairs to compute the transformation gives more accurate alignment parameters than directly using the peak parameters. In cases where a rigid transformation matrix cannot be reliably obtained, the voting is repeated inside a 
neighborhood of the corresponding peak, but with a smaller bin. A peak is chosen from this refined Hough Transform space, and used as the alignment parameters.

\section{Similarity Measure}

For each of the 10 different alignments, a matching score between two fingerprints is computed by comparing minutiae and orientation fields. The maximum value of the 10 scores is chosen as the final matching score between the two fingerprints. The details for computing matching scores of minutiae and orientation field are given below.

To compute minutiae matching score under a given alignment, we first find the corresponding minutiae pairs (one in the latent, one in the rolled print). For this purpose, we align the minutiae sets of the two fingerprints and then find an one-toone matching ${ }^{3}$ between the two minutiae sets using a greedy algorithm. For each minutia $m_{l}$ in the latent, a set of candidate minutiae in the rolled print is found. A minutia $m_{r}$ in the rolled print is called a candidate if it has not yet been matched to any minutia, and both its location and angle are sufficiently close to $m_{l}$. The threshold values $T_{\mathrm{S}}$ for spatial distance and $T_{\mathrm{A}}$ for angle distance were determined empirically. Among all candidates, the one closest to $m_{l}$ in location is chosen as the matching minutia of $m_{l}$.

After the corresponding minutiae are found, we compute a matching score between the latent and the rolled print. Suppose that $n$ pairs of matching minutiae between the latent and the rolled print are found. The minutiae matching score $S_{\mathrm{M}}$ between the two fingerprints is given by

$$
S_{\mathrm{M}}=\frac{1}{N} \sum_{i=1}^{n} s_{\mathrm{C}}(i) s_{\mathrm{S}}(i),
$$

where $s_{\mathrm{C}}(i)$ denotes the similarity between the minutia cylinder codes of the $i$ th pair of matched minutiae, $s_{\mathrm{S}}(i)=1-\frac{d_{\mathrm{S}}(i)}{2 T_{\mathrm{S}}}$ maps the spatial distance $d_{\mathrm{S}}(i)$ of the $i$ th pair of matched minutiae into a similarity score, and $N$ denotes the number of minutiae in the latent.

According to equation (4), the matching score depends on the number of matching minutiae, which itself is affected by the distance threshold $T_{\mathrm{S}}$. However, due to large distortion present in many latents, it is difficult to choose an appropriate value for $T_{\mathrm{S}}$. While a large threshold value will lead to more matching minutiae for distorted mated pairs, the number of matching minutiae for non-mated pairs will increase too. Hence, we use two different values (15 pixels and 25 pixels) for $T_{\mathrm{S}}$ and for each threshold, a set of matching minutiae is found and a matching score is computed using equation (4). The mean of the two scores is used as the minutiae matching score. Fig. 6 shows an example in which the score of the genuine pair is slightly reduced when the smaller threshold is used compared to the larger threshold, while the score of the latent and the rank-1 non-mate ${ }^{4}$ using large threshold is greatly reduced when the smaller threshold is used.

\footnotetext{
${ }^{3}$ One-to-one matching means that each minutia in the latent is matched to at most one minutia in the rolled print, and vice versa.

${ }^{4}$ The rank-1 non-mate refers to the non-mated rolled print whose match score with the latent ranks first among all rolled prints in the database.
}

We use a simple orientation field matcher that basically measures the consistency of the orientation differences. If we use Euclidean distance, for example, to measure the orientation differences, a small error in the rotation will contribute a small amount to the orientation difference for every block being compared, resulting in a large overall difference or small similarity score. In [36], the authors proposed a distance measure for orientation field matching that can handle small rotation errors. Given the aligned latent orientation field $O_{\mathrm{L}}$ and the rolled orientation field $O_{\mathrm{R}}$, each containing $K$ blocks, namely $O_{\mathrm{L}}(k)$ and $O_{\mathrm{R}}(k)$, the similarity between the two orientation fields is given by

$$
S_{\mathrm{O}}=\frac{\left|\sum_{k=1}^{K} v_{k} e^{j 2\left(O_{\mathrm{L}}(k)-O_{\mathrm{R}}(k)\right)}\right|}{\sum_{k=1}^{K} v_{k}},
$$

where $v_{k}$ is 1 if both corresponding blocks $k$ are valid, and 0 otherwise.

The overall matching score is given by

$$
S=\left(1-w_{\mathrm{O}}\right) S_{\mathrm{M}}+w_{\mathrm{O}} S_{\mathrm{O}},
$$

where the weight $w_{\mathrm{O}}$ is empirically set as 0.4 . Fig. 7 shows one example in which the fusion of minutiae matching and orientation field matching scores helps improve the retrieval rank $^{5}$ of the true mate. The retrieval rank of the true mate improved from 2 to 1 after the fusion, while the retrieval rank of the rank-1 non-mate according to minutiae matcher was changed from 1 to 3 after the fusion.

\section{EXPERIMENTAL RESULTS}

In this section, we first provide a description of the two databases used in our experiments, and the algorithms to be compared with the proposed algorithm. Then we report the performances of alignment and matching. This is followed by the fusion of matchers and the effect of fingerprint quality. Finally, we discuss the issue of computational cost.

\section{A. Latent Databases}

Matching experiments were conducted on two different latent fingerprint databases: NIST Special Database 27 (NIST SD27) and West Virginia University Latent Fingerprint Database (WVU LFD).

1) NIST Special Database 27 (NIST SD27): NIST Special Database 27 is the only publicly available database comprising latent fingerprints from operational scenarios (latents collected at crime scenes). It consists of 258 latent fingerprint images and 258 corresponding (mated) rolled prints. Both latents and rolled prints are available at 500 ppi. The quality of the latents in NIST SD27 varies, reflecting the operational (casework) quality.

NIST SD27 contains latent prints of three different qualities, termed "good", "bad", and "ugly", which were classified by latent examiners. Some examples of latents from those three qualities are shown in Fig. 2. Although this classification of

\footnotetext{
${ }^{5}$ Retrieval rank of a rolled fingerprint refers to its rank in the whole candidate list which is sorted in the decreasing order of matching score with the latent.
} 


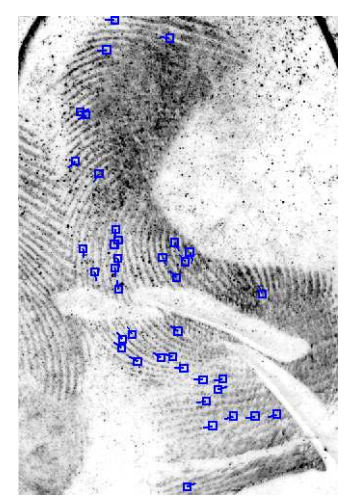

(a)

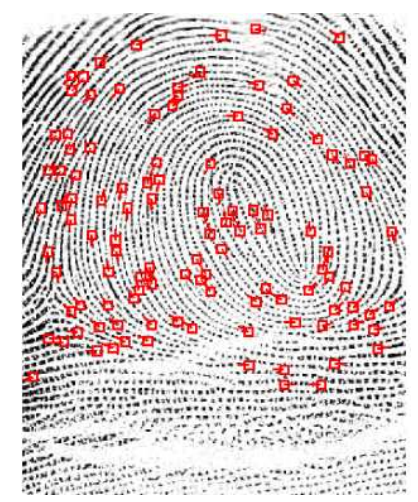

(b)

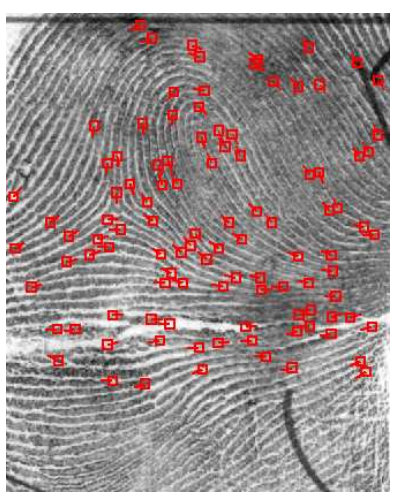

(c)

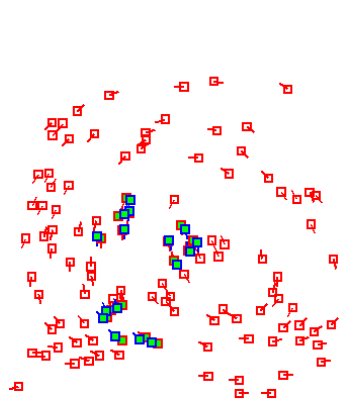

(d) 0.152 (e) 0.171

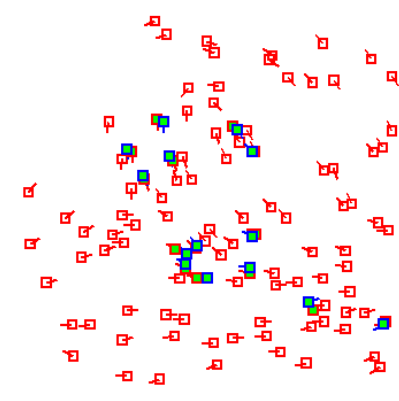

(f) 0.113

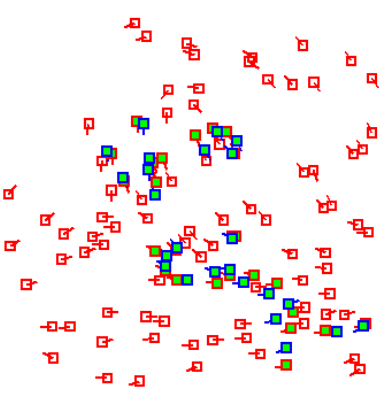

(g) 0.172

Fig. 6. Latent print in which the matching score of the genuine pair is slightly reduced when small threshold value is used compared to large threshold value, while impostor score is greatly reduced. (a)-(c) shows the latent, the true mate, and the rank-1 non-mate according to large threshold, respectively. (d)-(g) shows latent minutiae that were matched to rolled print minutiae in the following cases: (d) true mate using small threshold, (e) true mate using large threshold, (f) non-mate using small threshold, and (g) non-mate using large threshold. In (d)-(g), the scores corresponding to each case are included.

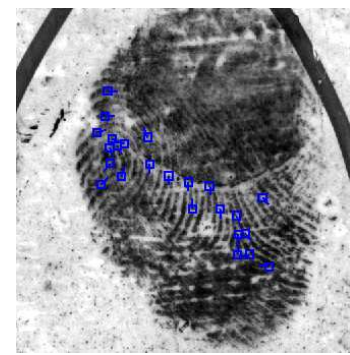

(a)

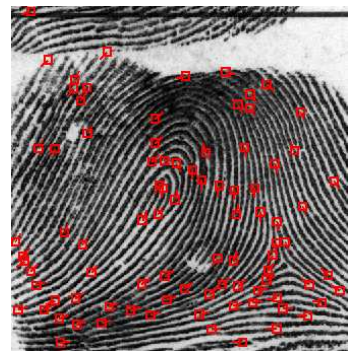

(b)

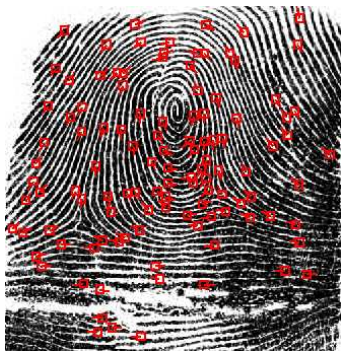

(c)

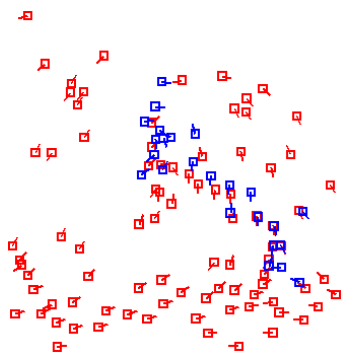

(d)

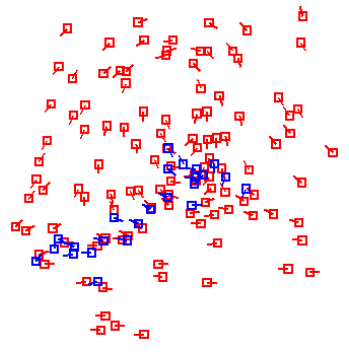

(e)

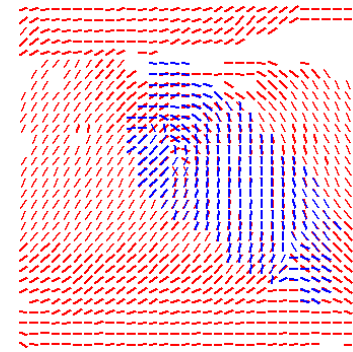

(f)

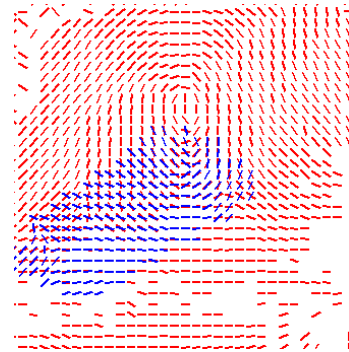

(g)

Fig. 7. Latent print identified at a higher rank after fusing minutiae matching scores with orientation field matching scores. The rank of the true mate was improved from 2 to 1 after the fusion, and the rank of the highest ranked non-mate was 3 after the fusion. (a)-(c) show minutiae and the image of (a) a latent, (b) its true mate, and (c) the highest ranked non-mate according to minutiae matching. (d) and (f) show latent minutiae and orientation field (in blue) aligned with minutiae and orientation field of the true mate. (e) and (g) show latent minutiae and orientation field (in blue) aligned with minutiae and orientation field of the rank-1 non-mate. 


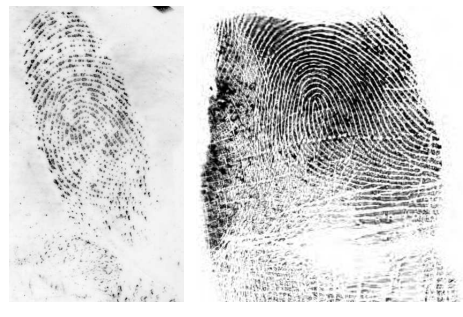

Fig. 8. A latent and its corresponding rolled print in WVU latent database The NFIQ quality of the rolled print is 4 .

latent prints as "good", "bad", and "ugly" is subjective, it has been shown that such a classification is correlated with the matching performance [15].

Another indicator of fingerprint quality that affects the matching performance is the number of minutiae in the latent print [15]. Based on the number of minutiae $n$ in latents in NIST SD27, Jain and Feng [15] classified latents in NIST SD27 into three groups: large $(n>21)$, medium $(13<n<$ 22 ), and small ( $n \leq 13)$, containing 86,85 , and 87 prints, respectively. We present our experimental results for each of the six quality groups based on subjective quality and the number of minutiae.

We use manually marked minutiae -provided with NIST SD27 - as features in latent fingerprints. For rolled fingerprint images, the minutiae are automatically extracted using the two commercial matchers.

2) West Virginia University Latent Database (WVU LFD): West Virginia University Latent Database ${ }^{6}$ consists of 449 latent fingerprint images collected in a laboratory environment and 4,740 rolled prints, including the 449 mated rolled prints of the 449 latents. The latent images in this database are at $1000 \mathrm{ppi}$, and they were converted to $500 \mathrm{ppi}$ for our experiments. Fig. 8 shows a latent with its corresponding rolled print in the WVU latent database. Manually marked minutiae were provided with these latents. Minutiae were automatically extracted from the rolled prints using the two commercial matchers.

There is no subjective quality value assigned to the latents in the WVU database. One of the objective quality measure depends on the number of minutiae in the latent, so any latent can be assigned an objective quality. If we apply the same objective quality classification scheme as in NIST SD27 to WVU database, we obtain 208, 80, and 161 latent fingerprints in the objective qualities of large, medium, and small number of minutiae, respectively.

The two latent databases, NIST SD27 and WVU, have different characteristics: most of the latent images in NIST SD27 contain significant background noise, while in WVU latent images, there is a uniform background in most latents. However, overall, the quality of the rolled prints in WVU database is worse than the quality of rolled prints in NIST SD27. This could be explained because in the operational database such as NIST SD27, rolled prints were captured by

\footnotetext{
${ }^{6}$ To request WVU latent fingerprint database, please contact Dr. Arun A. Ross (http://www.csee.wvu.edu/ ross/) at Integrated Pattern Recognition and Biometrics Lab (http://www.csee.wvu.edu/ ross/i-probe/).
}

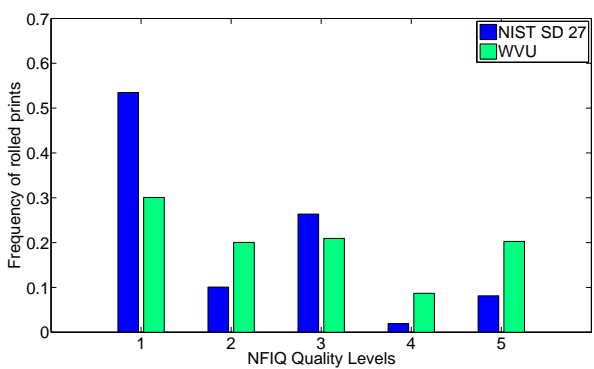

Fig. 9. Histograms of NFIQ values of rolled prints in NIST SD27 and WVU databases.

experienced law enforcement officers which may not be the case for the WVU database. If the rolled prints corresponding to the latents are of poor quality, the number of mated minutiae is small and, therefore, it is much more challenging to identify the mates of the latents at rank-1. Fig. 9 shows the histograms of NFIQ quality [37] of the rolled prints which have corresponding latents in NIST SD27 and in WVU databases (258 and 449 rolled prints, respectively). NFIQ defines five quality levels in the range $[1,5]$ with 1 indicating the highest quality.

\section{B. Commercial Matchers}

In order to compare the performance of the proposed latent fingerprint matcher, we used two commercial fingerprint matchers, referred to as COTS1 and COTS2. In addition, we also used the algorithm presented in [6], [38] as a benchmark, for which the SDK was provided by the authors (MCC SDK). It should be pointed out that none of the three matchers were designed specifically for the latent matching case. But, despite our efforts, we could not find any latent fingerprint matcher SDK or a forensic AFIS that is available for evaluation purposes by a research lab. Still, the matchers we are using in our comparative study are well known: one of the COTS (VeriFinger) [39] has been widely used as a benchmark in fingerprint publications, and MCC is one of the best performing algorithms in FVC-onGoing [40].

\section{Alignment Performance}

In order to estimate the alignment error, we use ground truth mated minutiae pairs from NIST SD27, which are marked by fingerprint examiners, to compute the average distance between the true mated pairs after alignment ${ }^{7}$. If the average Euclidean distance for a given latent is less than a pre-specified number of pixels in at least one of the ten best alignments (peaks in the Descriptor-Based Hough Transform), then we consider it a correct alignment. This alignment performance is shown in Fig. 10 for the NIST SD27 latent database. The xaxis shows the misalignment threshold ${ }^{8}$, and the $y$-axis shows

\footnotetext{
${ }^{7}$ Here we use the term ground truth minutiae to refer to minutiae which are marked by latent examiners by looking at the latent and the corresponding rolled print at the same time, and we use the term manually marked minutiae to refer to minutiae which are also marked in the latent by latent examiners, but without looking at the true mate (rolled print).

${ }^{8}$ The alignment is deemed as incorrect if the average distance between mated minutiae pairs after alignment is larger than this threshold.
} 


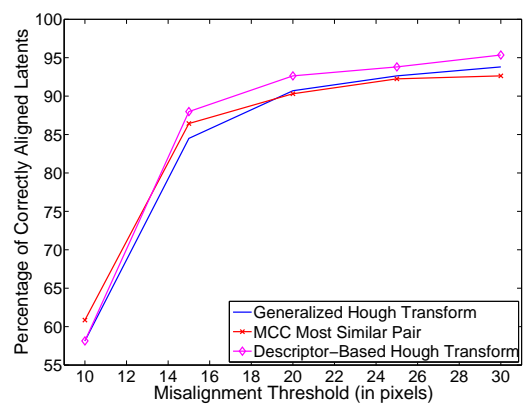

Fig. 10. Alignment Accuracy: percentage of correctly aligned latents vs. misalignment threshold.

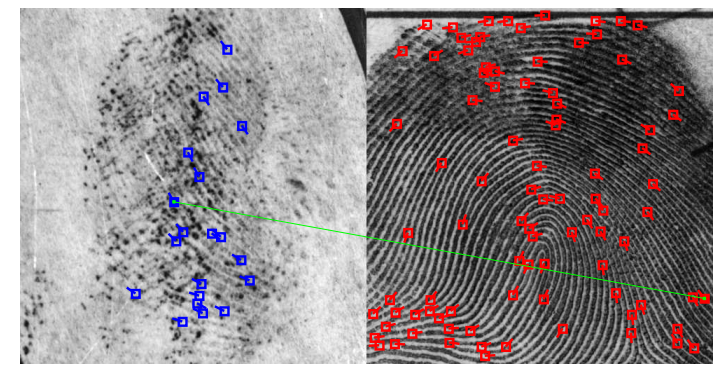

Fig. 12. Example of alignment error due to the small number of true mated minutia pairs in the overlapping area between a latent and its mated rolled print. Note that there is only one aligned minutiae pair here.

the percentage of correctly aligned latent fingerprints in at least one of the ten top alignments. For comparison, we show the accuracy of aligning the minutiae sets based on the peaks of the Generalized Hough Transform (GHT) and based on the most similar minutiae pair (according to the MCC similarity) Two latent alignment examples are given in Fig. 11 to show the alignment results by DBHT and GHT. As we can see from this figure, the proposed algorithm is superior to GHT in challenging cases where the number of minutiae is small.

There are very few errors in alignment if we set the threshold value of misalignment as 20 pixels. One of the reasons for these failure cases is there are a very small number of true mated minutia pairs in the overlapping area between the latent and mated rolled print. As a result, not many true mated pairs vote for the correct alignment parameters. The absence of true mated pairs is due to a limited number of minutiae in latents and the error in minutiae detection in the rolled print. One such example is shown in Fig. 12. Blue squares represent manually marked minutiae in the latent print (left), red squares represent automatically extracted minutiae in the rolled print (right), and the green line indicates the only true mated minutiae pair available for this (latent, rolled) image pair.

\section{Matching Performance}

In the identification scenario, the size of the background database (or gallery) significantly affects the identification accuracy. Therefore, to make the problem more challenging

\footnotetext{
${ }^{9}$ In this case, each alignment is based on one of the ten most similar minutiae pairs.
}

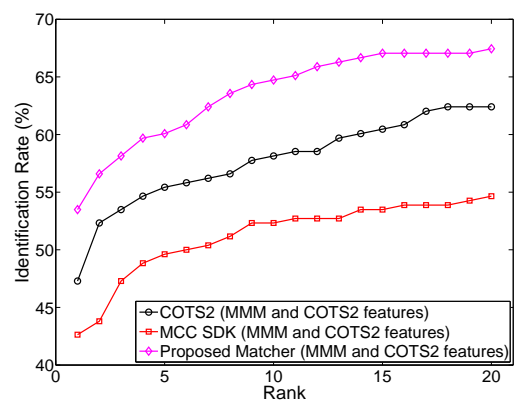

(a) NIST SD27

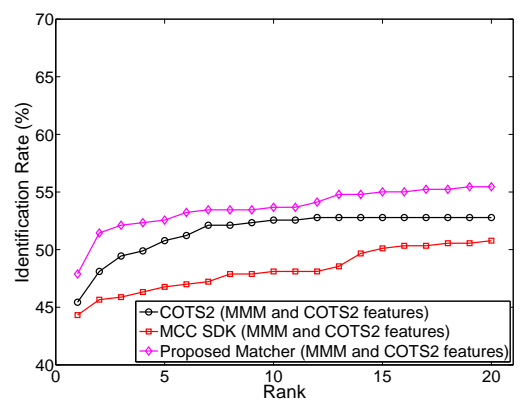

(b) WVU LFD

Fig. 13. Performance of COTS2, MCC SDK, and Proposed Matcher when the union of manually marked minutiae (MMM) extracted from latents and automatically extracted minutiae by COTS 2 from rolled prints is input to the matchers.

and realistic, we built a large background database of rolled prints by including the 258 mated rolled prints from NIST SD27, the 4,740 rolled prints from WVU database, and we added 27,000 rolled prints from the NIST Special Database 14 [41]. Therefore, the total number of rolled prints in the background database is 31,998 from a combination of the rolled prints in the three databases.

Minutia Cylinder Code (MCC) is used as local descriptor for minutiae in our experiments. The local descriptors are built using MCC SDK, which uses the bit-based implementation (binary descriptors)[38]. The parameters used for MCC are set as suggested in [38], with the number of cells along the cylinder diameter as $16\left(N_{s}\right)$. In our method, the local descriptor similarities are used in both the alignment and scoring process, as described in Section III.

Our matcher and MCC SDK take minutiae as input. In the latent cases, we use manually marked minutiae. For the rolled prints, we used both the COTS to extract minutiae. The performance of the proposed matcher using minutiae extracted from rolled prints using COTS2 is slightly worse on the NIST SD27 database compared to the performance using minutiae extracted using COTS1; however, for WVU LFD, using COTS2 minutiae yielded a significantly better performance compared to the performance using minutiae extracted using COTS1. This demonstrates that the performance of COTS can be significantly affected by the image quality. Overall, since minutiae extracted from COTS2 resulted in a better performance, we only report the results in which minutiae are extracted using COTS2. Fig. 13 shows the performance 

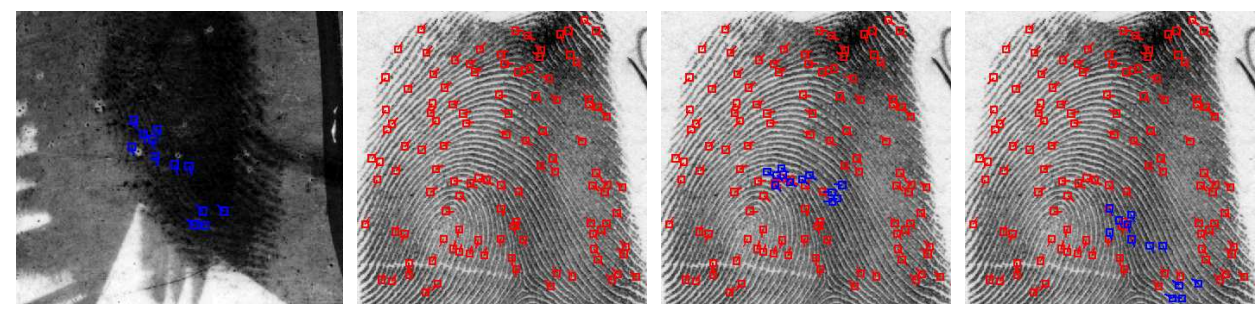

(a)
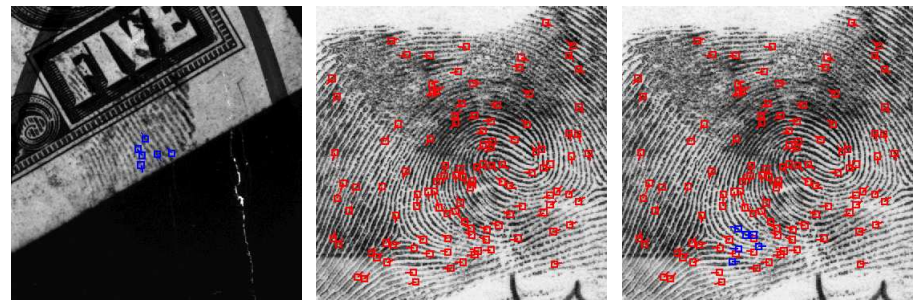

(b)

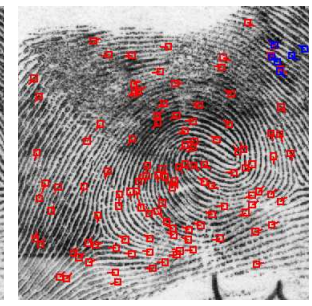

Fig. 11. Two examples in which DBHT (Descriptor-Based Hough Transform) alignment is better than GHT (Generalized Hough Transform) alignment. From left to right, latent with manually marked minutiae, corresponding rolled print with automatically extracted minutiae, rolled print with latent minutiae aligned by GHT, and aligned by DBHT.

of COTS2, MCC SDK, and the proposed matcher using manually marked minutiae in latents and automatically extracted minutiae by COTS2 in rolled prints. The proposed approach outperforms the other fingerprint matchers used in our study.

It is worth noticing that the matching performance on WVU LFD when manually marked minutiae are used is generally worse than the performance on NIST SD27. We believe this is due to a number of factors: (i) there are 14 latents with less than 3 manually marked minutiae in WVU LFD, while the minimum number of manually marked minutiae in NIST SD27 latents is 5; (ii) while the genuine (latent, rolled) pairs were provided with the database, after we examined the images in the WVU database we identified some that appeared to be wrongly paired; (iii) the quality of the mates (rolled prints) is slightly worse in WVU LFD than in NIST SD27. We did not exclude any of the latents or (latent, rolled) mated pairs from the WVU database (from cases (i) and (ii)) to allow future comparisons by other researchers with our results.

The performance of the COTS matchers, each using its own proprietary templates for latents (including automatically extracted minutiae and possibly other features), is worse than using manually marked minutiae for both the databases. However, the gap between the performances of manually marked minutiae and of proprietary template is much larger in the case of NIST SD27 than in the case of WVU latent database. This is probably due to the characteristics of the database. Note that WVU is a laboratory collected database and so most of the latents in it do not contain background noise. On the other hand, in NIST SD27 the images are of operational casework quality and most of the latents contain a large amount of background noise, which poses a challenge in automatic feature extraction. Fig. 14 shows the performance of the two COTS matchers using both manually marked minutiae and proprietary templates (automatically extracted minutiae) for NIST SD27 and WVU databases.

There have been several studies on latent matching reported

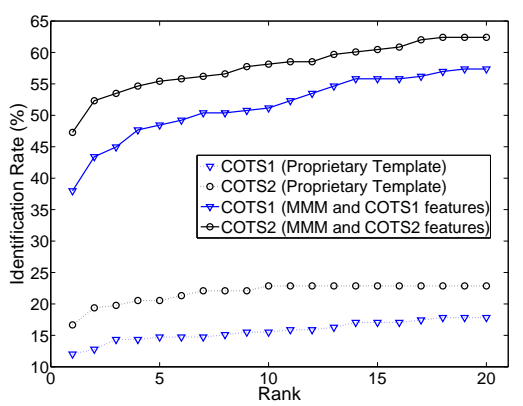

(a) NIST SD27

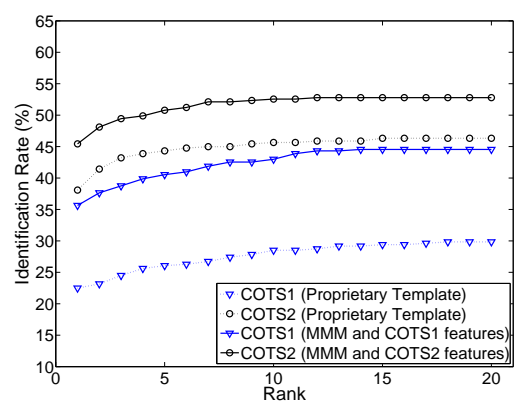

(b) WVU LFD

Fig. 14. Performance comparison using manually marked minutiae (MMM) and automatically extracted minutiae from latents.

in the literature. Almost all of them are based on NIST SD27. Table I shows most of the reported results on the matching performance for NIST SD27 database. There is no reported performance on the WVU latent database. It should be noticed that most of the reported results cannot be directly compared mainly because of two factors: (i) the amount of input information related to the latent fingerprint, which could be automatically extracted features, or manually marked 
TABLE I

COMPARISON OF RANK-1 ACCURACIES REPORTED IN THE LITERATURE FOR THE NIST SD27 DATABASE.

\begin{tabular}{|l|l|l|l|}
\hline Work & Manual Input & $\begin{array}{l}\text { Background } \\
\text { Size }\end{array}$ & $\begin{array}{l}\text { Rank-1 Ac- } \\
\text { curacy (\%) }\end{array}$ \\
\hline Jain and Feng [15] & $\begin{array}{l}\text { Minutiae, } \\
\text { Skeleton, SP, } \\
\text { ROI, RQM, } \\
\text { RFM, RWM }\end{array}$ & 29,257 & 74.0 \\
\hline $\begin{array}{l}\text { Proposed Matcher } \\
+ \text { COTS2 }\end{array}$ & Minutiae & 31,998 & 57.4 \\
\hline Proposed Matcher & Minutiae & 31,998 & 53.5 \\
\hline Paulino et al. [42] & $\begin{array}{l}\text { Minutiae, SP, } \\
\text { ROI }^{10}\end{array}$ & 27,258 & 48.0 \\
\hline Jain and Feng [15] & Minutiae & 29,257 & 34.9 \\
\hline Yoon et al. $[20]$ & SP, ROI ${ }^{10}$ & 27,258 & 26.0 \\
\hline
\end{tabular}

features such as minutiae, singular points, quality map, etc, or a combination of both; and (ii) some differences in the composition of the background databases and their size. In Table I we show the reported rank-1 accuracy, the manual input (for latents) used in each method, and the size of the background database used. One of the results that could be almost directly compared to our results is the reported rank1 accuracy (34.9\%) in [15] when only manually marked minutiae is used as input, which is the same scenario as in our proposed matcher. The proposed matcher achieves a significantly higher rank-1 accuracy of $53.5 \%$ with similar background database size and images as in [15].

Fig. 15 shows examples of latent prints in WVU LFD correctly identified at rank-1 by the proposed matcher. Even though the number of minutiae in the latents is small, they could still be correctly identified. The ranks of the true mates using COTS2 matcher are 1871 and 181, respectively.

Fig. 16 shows examples of latent prints in NIST SD27 and in WVU LFD whose mated full prints are not included in the top 20 candidates by the proposed matcher, but were correctly identified at rank- 1 by COTS 2 matcher. The ranks of these latents using the proposed matcher are 3626 and 64, respectively. In the first latent, a large number of minutiae do not have mated minutiae due to missing minutiae in the rolled print, and therefore the score is not as high as for impostor pairs in which many more minutiae could be matched. In the second case, we can see that the minutiae marked in the latent are relatively sparse, while the minutiae automatically extracted in the rolled print are denser. These facts make local neighborhoods (and descriptors) very different between the latent and its true mate, leading to a low match score.

\section{E. Fusion of Matchers}

We noticed that the two most accurate matchers (the proposed and COTS2) perform differently on different latents, meaning they are complementary to each other. This suggests that a fusion of these two matchers would result in a better performance. We performed a score-level fusion of these two matchers. The scores from COTS2 matcher were normalized to the range $[0,1]$ for each latent (local min-max normalization)

\footnotetext{
${ }^{10} \mathrm{SP}$ : singular points, ROI: region of interest, RQM: ridge quality map, RFM: ridge flow map, RWM: ridge wavelength map.
}

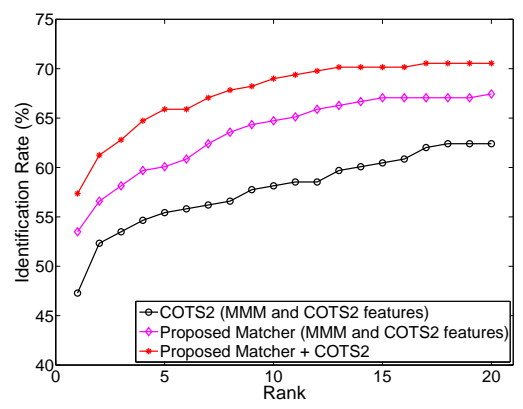

(a) NIST SD27

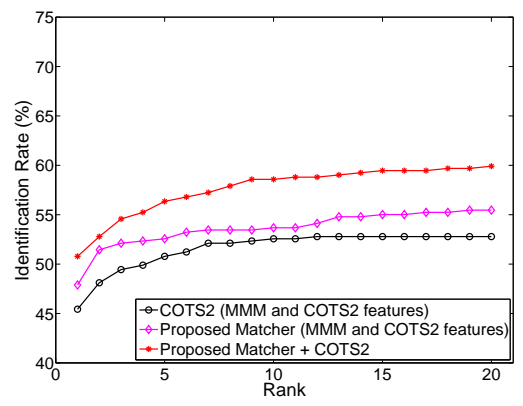

(b) WVU LFD

Fig. 17. Score-level fusion of the proposed matcher and COTS2 for NIST SD27 and WVU databases.

because local normalization was shown to perform better than global normalization in the identification scenario [43]. Although the proposed matcher and COTS2 matcher have similar strength, the fusion weights selected $(0.8$ and 0.2$)$ were not equal because of the large range of the scores for the COTS2 matcher. The performance improvement obtained by the score-level fusion of COTS2 matcher and the proposed matcher is shown in Fig. 17 for both the databases. Some examples in which the fusion of the two matchers (COTS2 and proposed matcher) improved the ranks of the true mates compared to the retrieval ranks by the individual matchers separately are shown in Fig. 18 and 19. Note that like those mated pairs (shown in Fig. 15 and Fig. 16) identified at rank-1 by either one the two matchers, mated pairs (shown in Fig. 18 and Fig. 19) which both matchers failed to identify at rank1 also benefit from the fusion. The reason is the scores of non-mated pairs given by the two matchers are not consistent.

Improvements were also obtained by combining the proposed matcher and other matchers in our study (COTS1 and MCC SDK), but they are not reported here because the fusion performance with COTS2 was consistently better than the performance of COTS1 and of MCC SDK. We also performed rank-level fusion using the highest rank and Borda Count methods [44]. However, since score-level fusion showed a better performance, we only report here results for score-level fusion.

\section{F. Effect of Fingerprint Quality}

In Section IV-A, we discuss how the quality of the latent fingerprints can be measured subjectively (assigned by latent 

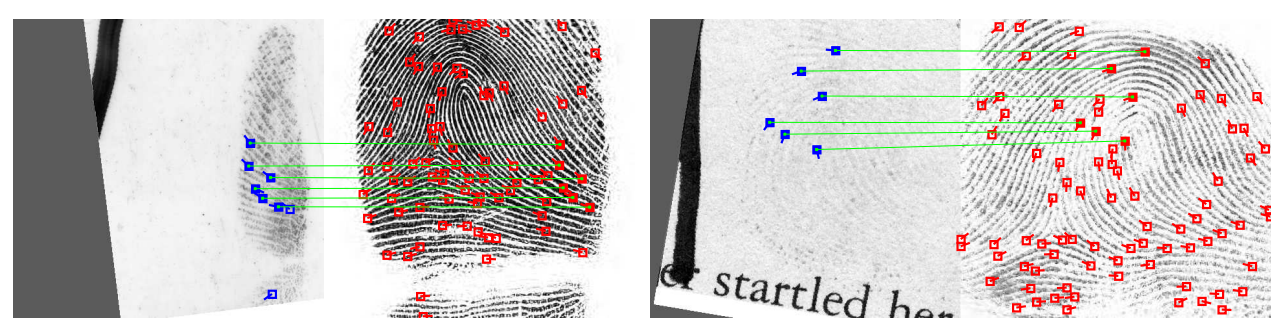

Fig. 15. Latent prints correctly identified at rank-1 by the proposed matcher but ranked below 20 by COTS2.
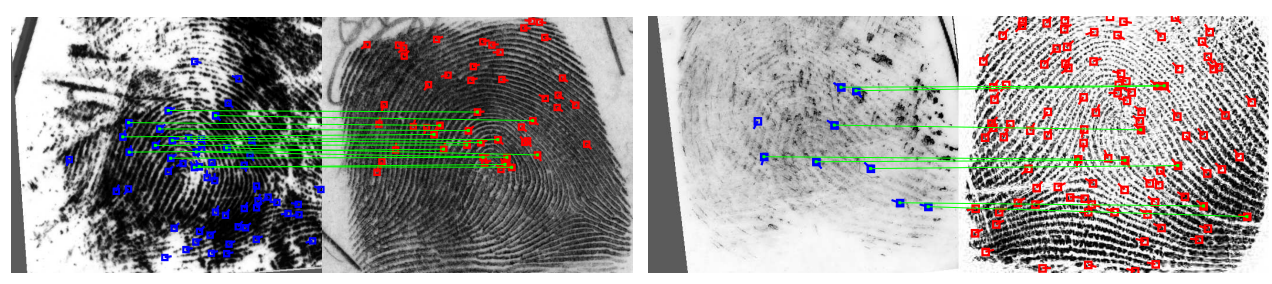

Fig. 16. Latent prints whose mates were not retrieved in the top 20 candidates by the proposed matcher but correctly identified at rank-1 by COTS2 matcher.

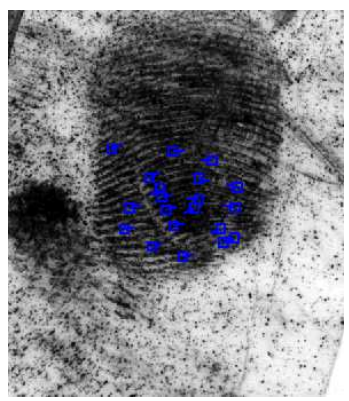

(a)

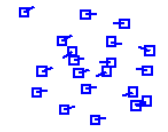

(e)

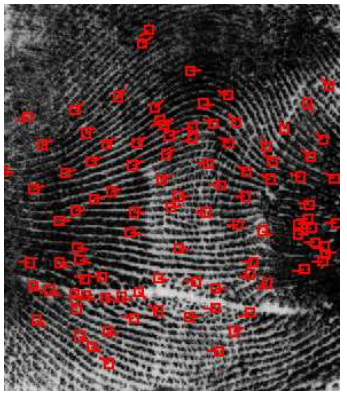

(b) $(5,10)$

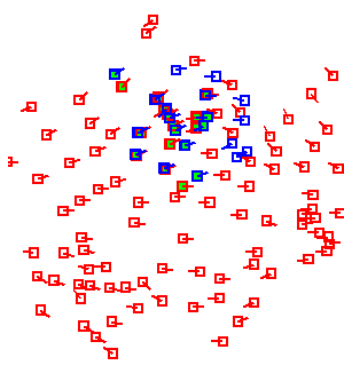

(f)

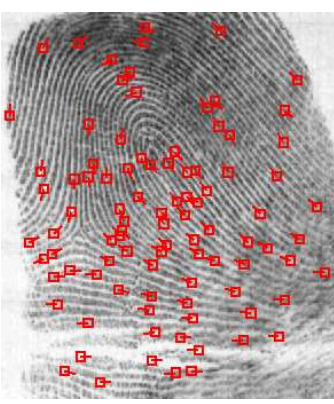

(c) $(1,30848)$

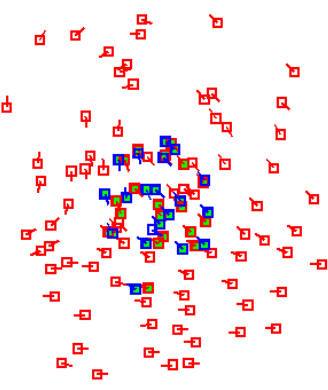

(g)

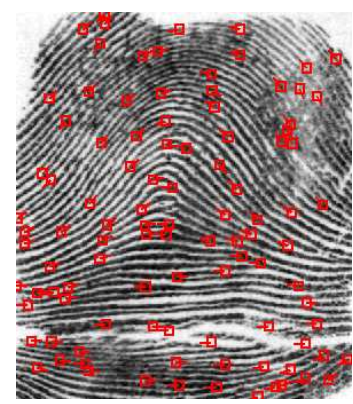

(d) $(3617,1)$

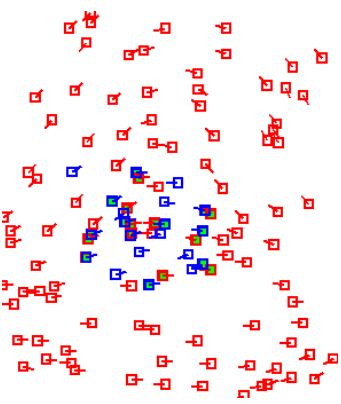

(h)

Fig. 18. Latent print mate from NIST SD 27 identified at rank 1 after score-level fusion of COTS2 and proposed matcher. The first row shows (a) a latent, (b) its true mate, (c) rank-1 non-mate by the proposed matcher, and (d) rank-1 non-mate by COTS2 matcher. The second row shows (e) latent minutiae, (f)-(h) latent minutiae (in blue) aligned by the proposed matcher to the rolled print minutiae shown in (b)-(d). In (b)-(d), the numbers in parentheses indicate the ranks that each rolled print was retrieved by the proposed matcher and COTS2 matcher, respectively.

experts as in NIST SD27) and objectively (based on the number of minutiae available). Rank-1 accuracies are shown for each quality separately in Tables II, III, and IV for both the latent databases. We can see that the matching performance is highly correlated with the number of minutiae available in the latent prints. The performance of the proposed matcher is consistently better over all qualities and for both the databases.

The quality of full prints also has a large impact on the matching accuracy. In Fig. 9, the histograms of NFIQ quality values for the corresponding rolled prints in each latent database are shown. According to the NFIQ quality measure,
TABLE II

RANK-1 ACCURACIES FOR VARIOUS SUBJECTIVE QUALITY LEVELS OF LATENTS IN NIST SD27.

\begin{tabular}{|c|c|c|c|c|}
\hline Quality & COTS1 (\%) & COTS2 (\%) & MCC (\%) & Proposed (\%) \\
\hline All & 38.0 & 47.3 & 42.6 & 53.5 \\
\hline Good & 55.7 & 70.5 & 69.3 & 75.0 \\
\hline Bad & 36.5 & 36.5 & 31.8 & 47.1 \\
\hline Ugly & 21.2 & 34.1 & 25.9 & 37.6 \\
\hline
\end{tabular}

the quality of the rolled prints in WVU database is slightly worse than the quality of the rolled prints in NIST SD27. The NFIQ quality measure is an integer value in the range 1 to 5 , 


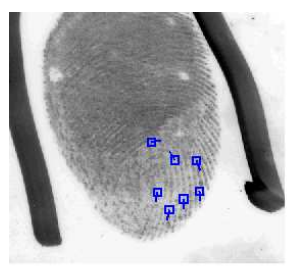

(a)

$$
\text { ㅁ. }
$$

(e)

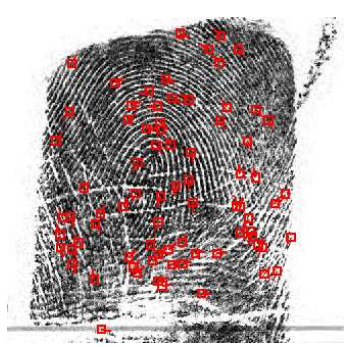

(b) $(2,42)$

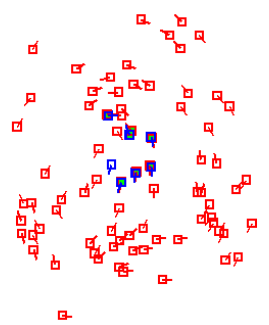

(f)

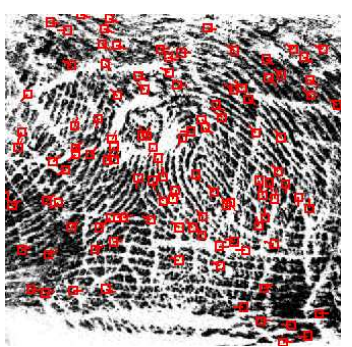

(c) $(1,29408)$

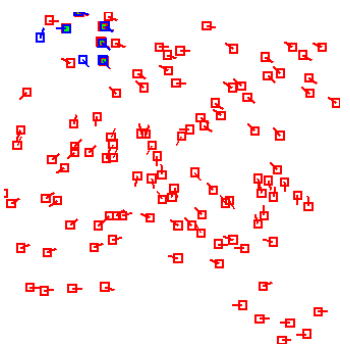

(g)

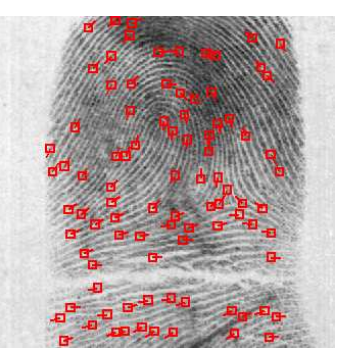

(d) $(48,1)$

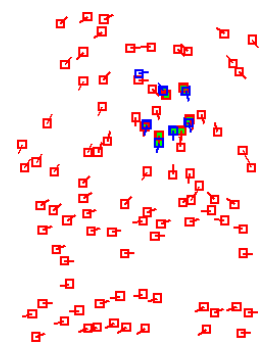

(h)

Fig. 19. Latent print mate from WVU LFD identified at rank 1 after score-level fusion of COTS2 and proposed matcher. The first row shows (a) a latent, (b) its true mate, (c) rank-1 non-mate by the proposed matcher, and (d) rank-1 non-mate by COTS2 matcher. The second row shows (e) latent minutiae, (f)-(h) latent minutiae (in blue) aligned by the proposed matcher to the rolled print minutiae shown in (b)-(d). In (b)-(d), the numbers in parentheses indicate the ranks that each rolled print was retrieved by the proposed matcher and COTS2 matcher, respectively.

TABLE III

RANK-1 ACCURACIES FOR VARIOUS OBJECTIVE QUALITY VALUES OF LATENTS IN NIST SD27 (LARGE, MEDIUM AND SMALL REFER TO THE NUMBER OF MINUTIAE IN THE LATENT).

\begin{tabular}{|c|c|c|c|c|}
\hline Quality & COTS1 (\%) & COTS2 (\%) & MCC (\%) & Proposed (\%) \\
\hline All & 38.0 & 47.3 & 42.6 & 53.5 \\
\hline Large & 59.3 & 73.3 & 70.9 & 75.6 \\
\hline Medium & 43.5 & 45.9 & 43.5 & 56.5 \\
\hline Small & 11.5 & 23.0 & 13.8 & 28.7 \\
\hline
\end{tabular}

TABLE IV

RANK-1 ACCURACIES FOR VARIOUS OBJECTIVE QUALITY VALUES OF LATENTS IN WVU LFD (LARGE, MEDIUM AND SMALL REFER TO THE NUMBER OF MINUTIAE IN THE LATENT).

\begin{tabular}{|c|c|c|c|c|}
\hline Quality & COTS1 (\%) & COTS2 (\%) & MCC (\%) & Proposed (\%) \\
\hline All & 35.6 & 45.4 & 44.3 & 47.9 \\
\hline Large & 63.5 & 73.1 & 74.0 & 74.5 \\
\hline Medium & 28.8 & 45.0 & 37.5 & 45.0 \\
\hline Small & 3.1 & 9.9 & 9.3 & 14.9 \\
\hline
\end{tabular}

TABLE V

RANK-1 ACCURACIES FOR LATENTS GROUPED ACCORDING TO NFIQ QUALITY VALUES OF CORRESPONDING ROLLED PRINTS IN NIST SD27.

\begin{tabular}{|c|c|c|c|c|}
\hline Quality & COTS1 (\%) & COTS2 (\%) & MCC (\%) & Proposed (\%) \\
\hline All & 38.0 & 47.3 & 42.6 & 53.5 \\
\hline NFIQ $\leq 3$ & 42.1 & 54.9 & 49.4 & 60.4 \\
\hline NFIQ $>3$ & 30.9 & 34.0 & 30.9 & 41.5 \\
\hline
\end{tabular}

TABLE VI

RANK-1 ACCURACIES FOR LATENTS GROUPED ACCORDING TO NFIQ QUALITY VALUES OF CORRESPONDING ROLLED PRINTS IN WVU LFD.

\begin{tabular}{|c|c|c|c|c|}
\hline Quality & COTS1 (\%) & COTS2 (\%) & MCC (\%) & Proposed (\%) \\
\hline All & 35.6 & 45.4 & 44.3 & 47.9 \\
\hline NFIQ $\leq 3$ & 36.9 & 50.0 & 48.0 & 52.4 \\
\hline NFIQ $>3$ & 34.4 & 41.1 & 40.6 & 43.3 \\
\hline
\end{tabular}

where 1 is the highest quality and 5 is the worst quality. We observed a significant difference in the matching performance when the latents were divided into the following two quality groups: (i) rolled prints are of good quality (NFIQ value of 1,2 and 3), and (ii) rolled prints are of poor quality (NFIQ values of 4 and 5 ). The difference in matching performance between good NFIQ and poor NFIQ qualities for all matchers ranges from $11-21 \%$ for NIST SD27, while it ranges from $2-9 \%$ for WVU database (see Tables V and VI). As an example, the rank-1 accuracy of COTS2 matcher on NIST SD27 is $54.9 \%$ and $34.0 \%$ for good and poor NFIQ quality, respectively.

\section{G. Computational Cost}

The implementation of our matching algorithm is in Matlab. The speed of our matcher running in a PC with Intel Core 2 Quad CPU and Windows XP operating system is around 10 matches per second. Multi-thread capability was not utilized. The majority of the running time $(70 \%)$ is spent matching the local minutiae descriptors. In a $\mathrm{C} / \mathrm{C}++$ implementation, this matching would be much faster than in Matlab because of the nature of the MCC descriptors (binary). We did not spend time optimizing the code for speed.

\section{CONClusions AND Future Work}

We have presented a fingerprint matching algorithm designed for matching latents to rolled/plain fingerprints which is based on a descriptor-based Hough Transform alignment. A comparison between the alignment performance of the proposed algorithm and the well-known Generalized Hough Transform shows the superior performance of the proposed method. We also reported matching results for two different latent fingerprint databases with a large background database 
of around $32 \mathrm{~K}$ rolled prints. We compared the performance of the proposed matcher with three different state-of-theart fingerprint matchers. Experimental results show that the proposed algorithm performs better than the three fingerprint matchers used in the study across all image qualities. A score-level fusion of the proposed matcher and one of the commercial matchers (COTS2) shows a further boost in the matching performance.

We plan to include a texture-based descriptor to improve the matching accuracy especially when the overlap between the latent and rolled prints is small. This was suggested in [31]. In our future work, following the recommendations in [26], [15], we plan to include additional automatically extracted features to improve the matching performance without an increase in manual labor (latent examiner's markups). Although the proposed matcher is more accurate than the two COTS matchers, they are significantly faster. We also plan to develop an indexing algorithm to speed up latent matching.

\section{REFERENCES}

[1] A. A. Paulino, J. Feng, and A. K. Jain, "Latent fingerprint matching using descriptor-based Hough transform," in Int'l Joint Conf. on Biometrics, October 2011, pp. 1-7.

[2] C. Champod, C. Lennard, P. Margot, and M. Stoilovic, Fingerprints and Other Ridge Skin Impressions. CRC Press LLC, 2004.

[3] NIST Special Database 27, "Fingerprint minutiae from latent and matching tenprint images," http://www.nist.gov/srd/nistsd27.cfm.

[4] D. R. Ashbaugh, Quantitative-Qualitative Friction Ridge Analysis. CRC Press, 1999

[5] FBI, "Next generation identification," http://www.fbi.gov/aboutus/cjis/fingerprints_biometrics/ngi.

[6] R. Cappelli, M. Ferrara, and D. Maltoni, "Minutia cylinder-code: a new representation and matching technique for fingerprint recognition," IEEE Trans. on Pattern Analysis and Machine Intelligence, vol. 32, no. 12, pp. 2128-2141, December 2010.

[7] D. Maltoni, D. Maio, A. K. Jain, and S. Prabhakar, Handbook of Fingerprint Recognition, 2nd ed. Springer-Verlag, 2009.

[8] M. Tico and P. Kuosmanen, "Fingerprint matching using and orientationbased minutia descriptor," IEEE Trans. on Pattern Analysis and Machine Intelligence, vol. 25, no. 8, pp. 1009-1014, August 2003.

[9] J. Qi, S. Yang, and Y. Wang, "Fingerprint matching combining the global orientation field with minutia," Pattern Recognition Letters, vol. 26, pp. 2424-2430, 2005.

[10] J. Gu, J. Zhou, and C. Yang, "Fingerprint recognition by combining global structure and local cues," IEEE Trans. on Image Processing, vol. 15, no. 7, pp. 1952-1964, July 2006.

[11] X. Jiang and W.-Y. Yau, "Fingerprint minutiae matching based on the local and global structures," in Proc. $15^{\text {th }}$ Int'l Conf. Pattern Recognition, 2000, pp. 1038-1041.

[12] X. Chen, J. Tian, and X. Yang, "A new algorithm for distorted fingerprints matching based on normalized fuzzy similarity measure," IEEE Trans. on Image Processing, vol. 15, no. 3, pp. 767-776, March 2006.

[13] W. Xu, X. Chen, and J. Feng, "A robust fingerprint matching approach: Growing and fusing of local structures," in International Conference on Biometrics, Seoul, Korea, August 2007, pp. 134-143.

[14] J. Feng, "Combining minutiae descriptors for fingerprint matching," Pattern Recognition, vol. 41, pp. 342-352, 2008

[15] A. K. Jain and J. Feng, "Latent fingerprint matching," IEEE Trans. on Pattern Analysis and Machine Intelligence, vol. 33, no. 1, pp. 88-100, January 2011.

[16] J. Feng, S. Yoon, and A. K. Jain, "Latent fingerprint matching: Fusion of rolled and plain fingerprints," in International Conference on Biometrics (ICB), June 2009

[17] A. K. Jain, J. Feng, A. Nagar, and K. Nandakumar, "On matching latent fingerprints," in CVPR Workshops on Biometrics, June 2008, pp. 1-8.

[18] J. Feng and A. K. Jain, "Filtering large fingerprint database for latent matching," in $I C P R$, December 2008, pp. 1-4.

[19] S. Yoon, J. Feng, and A. K. Jain, "On latent fingerprint enhancement," in SPIE, vol. 7667, no. 766707, April 2010.
[20] — , "Latent fingerprint enhancement via robust orientation field estimation," in Int'l Joint Conf. on Biometrics, October 2011.

[21] A. Sankaran, T. I. Dhamecha, M. Vatsa, and R. Singh, "On matching latent to latent fingerprints," in Int'l Joint Conf. on Biometrics, October 2011, pp. 1-6.

[22] M. Vatsa, R. Singh, A. Noore, and K. Morris, "Simultaneous latent fingerprint recognition," Applied Soft Computing, vol. 11, no. 7, pp 4260-4266, October 2011.

[23] NIST, "Evaluation of latent fingerprint technologies," http://www.nist.gov/itl/iad/ig/latent.cfm.

[24] — "Summary of the results of Phase I ELFT testing," http://biometrics.nist.gov/cs_links/latent/elft07/phase1_aggregate.pdf, September 2007.

[25] M. Indovina, V. Dvornychenko, E. Tabassi, G. Quinn, P. Grother, S. Meagher, and M. Garris, "An evaluation of automated latent fingerprint identification technology (Phase II)," NIST Interagency/Internal Report (NISTIR) - 7577, Tech. Rep., April 2009.

[26] M. Indovina, R. A. Hicklin, and G. I. Kiebuzinski, "NIST evaluation of latent fingerprint technologies: Extended feature sets [Evaluation \#1]," NIST Interagency/Internal Report (NISTIR) - 7775, Tech. Rep., April 2011.

[27] B. T. Ulery, R. A. Hicklin, J. Buscaglia, and M. A. Roberts, "Accuracy and reliability of forensic latent fingerprint decisions," in Proc. of the National Academy of Sciences of the USA, vol. 108, no. 19, 2011, pp. 7733-7738.

[28] — " "Repeatability and reproducibility of decisions by latent fingerprint examiners," PLOS ONE, vol. 7, no. 3, p. e32800, March 2012.

[29] I. E. Dror, D. Charlton, and A. E. Peron, "Contextual information renders experts vulnerable to making erroneous identifications," Forensic Science International, vol. 156, no. 1, pp. 74-78, 2006.

[30] J. Feng and A. K. Jain, "Fingerprint reconstruction: from minutiae to phase," IEEE Trans. on Pattern Analysis and Machine Intelligence, vol. 33, no. 2, pp. 209-223, February 2011.

[31] J. Feng and J. Zhou, "A performance evaluation of fingerprint minutia descriptors," in Int'l Conf. on Hand-Based Biometrics, 2011, pp. 1-6.

[32] B. G. Sherlock and D. M. Monro, "A model for interpreting fingerprint topology," Pattern Recognition, vol. 26, no. 7, pp. 1047-1055, 1993.

[33] J. Zhou and J. Gu, "Modeling orientation fields of fingerprints with rational complex functions," Pattern Recognition, vol. 37, pp. 389-391, 2004.

[34] S. Huckemann, T. Hotz, and A. Munk, "Global models for the orientation field of fingerprints: an approach based on quadratic differentials," IEEE Trans. on Pattern Analysis and Machine Intelligence, vol. 30, no. 9, pp. 1507-1519, September 2008.

[35] N. K. Ratha, K. Karu, S. Chen, and A. K. Jain, "A real-time matching system for large fingerprint databases," IEEE Trans. on Pattern Analysis and Machine Intelligence, vol. 18, no. 8, pp. 799-813, August 1996.

[36] X. Jiang, M. Liu, and A. C. Kot, "Fingerprint retrieval for identification," IEEE Trans. on Information Forensics and Security, vol. 1, no. 4, pp. 532-542, December 2006.

[37] E. Tabassi, C. L. Wilson, and C. I. Watson, "NIST fingerprint image quality," NIST Interagency/Internal Report (NISTIR) - 7151, Tech. Rep., August 2004.

[38] R. Cappelli, M. Ferrara, D. Maltoni, and M. Tistarelli, "MCC: a baseline algorithm for fingerprint verification in FVC-onGoing," in $11^{\text {th }}$ Int. Conf. Control, Automation, Robotics and Vision, December 2010, pp. $19-23$.

[39] Neurotechnology Inc, "Verifinger," http://www.neurotechnology.com/ verifinger.html.

[40] Biometric System Laboratory team, "FVC-onGoing," https://biolab.csr.unibo.it/FVCOnGoing/UI/Form/Home.aspx.

[41] NIST Special Database 14, "Mated fingerprint card pairs 2," http://www.nist.gov/srd/nistsd14.cfm.

[42] A. A. Paulino, A. K. Jain, and J. Feng, "Latent fingerprint matching: Fusion of manually marked and derived minutiae," in 23rd SIBGRAPI Conference on Graphics, Patterns and Images, August 2010, pp. 63-70.

[43] V. N. Dvornychenko, "Evaluation of fusion methods for latent fingerprint matchers," in $I C B, 2012$, to Appear.

[44] A. A. Ross, K. Nandakumar, and A. K. Jain, Handbook of Multibiometrics. Springer, 2006 